\title{
How can clinical immunology laboratories contribute to the management of severe COVID-19 cases in limited resource contexts?
}

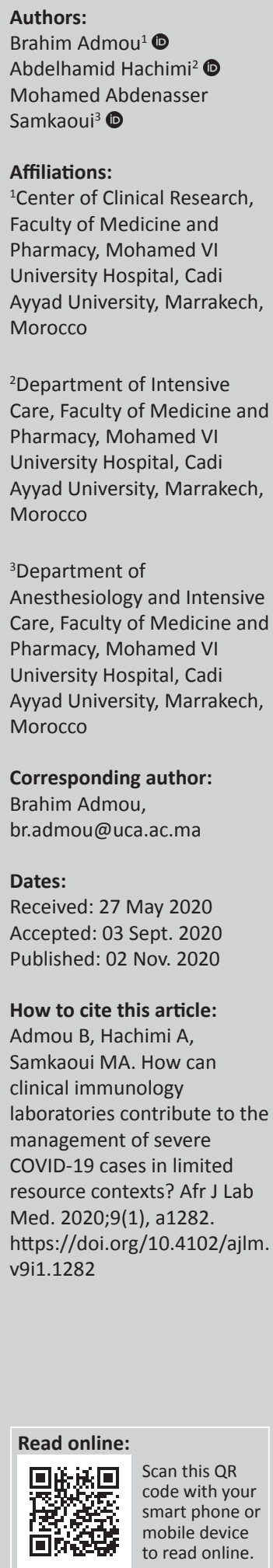

\section{Introduction}

Since its appearance, the coronavirus disease 2019 (COVID-19) that is caused by severe acute respiratory disease coronavirus 2 (SARS-CoV-2), has been responsible for severe respiratory disease similar to diseases commonly associated with the coronaviruses such as severe acute respiratory syndrome and Middle East respiratory syndrome; COVID-19 frequently requires hospitalisation in the intensive care unit and has had a high mortality rate. ${ }^{1}$ The severity of COVID-19 is generally stratified as asymptomatic, mild (without signs of severe or critical illness), severe (with signs of breathing difficulties) or critical (with signs of respiratory failure, shock and multiple organ failure). ${ }^{2}$

The contribution of the medical laboratory to the categorisation of COVID-19 clinical forms is not yet well defined. ${ }^{3}$ After confirmation of infection by real-time polymerase chain reaction, biochemical and haematological analyses provide fundamental biological parameters for determining disease severity. Similarly, the clinical immunology laboratory could play an important role in elucidating diverse immunological abnormalities associated with the disease. In particular, immunological testing could better categorise the severe and critical forms of COVID-19, and subsequently assist treating physicians during the entire course of therapy. The purpose of this opinion article is to highlight the position of the immunology laboratory in the management of severe and critical COVID-19 cases, specifically in countries with limited resources.

\section{COVID-19 and cytokine storm}

COVID-19 is marked by an overproduction of pro-inflammatory cytokines and chemokines, mainly interleukin (IL)-6, IL-10, tumor necrosis factor (TNF)- $\alpha$, IL-1 $\beta$, IL-2, IL-10, interferon- $\gamma$ and monocyte chemoattractant protein $-1{ }^{4}$ Other biological abnormalities may predict the severity or progression of the disease, such as abnormal coagulation activation, leukopenia, high levels of C-reactive protein, ferritin, D-dimers, aminotransferase, lactate dehydrogenase and creatin kinase. ${ }^{3,5}$ Elevated levels of pro-inflammatory cytokines have been shown to characterise severe lung infection, which manifests as respiratory distress, multiple organ failure and adverse outcomes in SARS-CoV-2 infection. ${ }^{6,7}$ In addition, IL-6, IL-10, and TNF- $\alpha$ levels significantly increase during infection and drop during recovery, ${ }^{8}$ suggesting that the intensity of the cytokine release correlates with disease activity. Interestingly, IL-6 may be a marker for monitoring serious COVID-19 cases. $^{9}$

Moreover, simultaneous high levels of IL-6 and D-dimers have been shown to be narrowly associated with severe forms of the disease in adult patients. Therefore, measuring them in combination allows for greater specificity and sensitivity for predicting severe cases of COVID-19. ${ }^{3}$

\section{COVID-19 and T cell impairment}

Deregulation of the immune response, particularly $\mathrm{T}$ lymphocytes, appears to be strongly linked to the pathology of COVID-1910; direct infection of lymphocytes by SARS-CoV-2 has been proposed as a cause for acute lymphocyte decline.,11 Lymphocytes express the receptor angiotensin-converting enzyme 2, which has been suggested to be the primary receptor targeted by SARS-CoV-2. ${ }^{12}$

Copyright: (C) 2020. The Authors. Licensee: AOSIS. This work is licensed under the Creative Commons Attribution License. 
Moreover, altered $\mathrm{T}$ lymphocytes could be an important factor in worsening symptoms in patients, which makes lymphopenia a relevant marker of disease severity, hence the need for intensive care unit admission. ${ }^{11}$

It has been demonstrated that the severe respiratory syndrome that manifests due to COVID-19 is characterised by cluster of differentiation (CD)4 and CD8 T lymphopenia, correlating with disease severity. ${ }^{4,13}$ On the other hand, COVID-19 patients who require intensive care unit hospitalisation have significantly higher levels of IL-6, IL-10 and TNF- $\alpha$, with lower levels of CD4 and CD8 T cell counts, ${ }^{8}$ with which levels of cytokines and $\mathrm{T}$ cells are inversely correlated. ${ }^{6,8}$ Moreover, because $\mathrm{T}$ lymphocytes are generally crucial for amortising exaggerated natural immune reactions against viral infection, $\mathrm{T}$ cell defects can lead to worsening inflammatory responses during COVID-19, whereas restoring the number of these lymphocytes can improve them. ${ }^{6}$

In accordance with this hypothesis, it has been shown that 4-6 days following the onset of infection, $\mathrm{T}$ lymphocyte counts drop to their lowest level, whereas cytokine levels reach their maximum. Conversely, the restoration of the number of $\mathrm{T}$ lymphocytes is associated with a decrease in serum levels of various cytokines, such as IL-6, IL-10 and TNF- $\alpha .{ }^{6}$ Owing to the cytokine storm, and other immunological predictors of severity of SARS-CoV2 infection, these may also be helpful in choosing antiinflammatory drugs, especially corticosteroids for their potential benefit in the reduction of inflammation-induced lung damage in severe COVID-19 patients. ${ }^{1}$

\section{COVID-19 and immunological investigations}

Having shown that the most relevant immunological parameters include inflammatory cytokines and $\mathrm{T}$ lymphocyte subpopulations, the clinical immunology laboratory is positioned to be important for assessing COVID-19 patients, especially for categorising, or even predicting severe cases. ${ }^{2,10}$ Cytokine levels, especially that of IL-6, can be individually measured on immunoassay analysers, whereas other cytokine profiles can be explored using either enzyme-linked immunosorbent assays or other specific biotechnologies like Luminex ${ }^{\circledast}, 200^{\mathrm{TM}}$ (or multiplex) (Luminex ${ }^{\circledR}$, Austin, Texas, United States) and flow cytometry systems, which allow for a large-scale quantitative measurement. ${ }^{14}$ The assessment of the main $\mathrm{T}$ cell subsets, such as $\mathrm{CD}^{+}, \mathrm{CD}^{+}$and $\mathrm{CD}^{+}$, requires only a simple phenotyping procedure conductible on a mini-cytometer, which is largely available worldwide even in limitedresource context laboratories, thanks to HIV management programs. More developed flow cytometry platforms allow for comprehensive phenotyping assays, enabling the investigation of naive, memory and regulatory $\mathrm{T}$ cells; in severe COVID-19 patients, early data on $\mathrm{T}$ cell subpopulation abnormalities show increased naive helper $\mathrm{T}$ cells and decreased memory helper T cells, associated with a lower number of regulatory T cells. ${ }^{10}$

Using a receiver operating characteristic curve to compare fatal and recovered COVID-19 cases, $\mathrm{Xu}$ et al. ${ }^{2}$ considered 559 cells $/ \mu \mathrm{L}, 235$ cells $/ \mu \mathrm{L}$, and 104 cells $/ \mu \mathrm{L}$ of $\mathrm{CD}^{+}, \mathrm{CD}^{+} \mathrm{T}$, and $\mathrm{CD}^{+} \mathrm{T}$ cell subsets as warning values, below which there was a significantly higher risk of in-hospital death. ${ }^{2}$ Indeed, there is a need for regional data analyses before determining cut-off values of these $\mathrm{T}$ cell subsets. However, T cell lymphopenia might be accentuated by possible primary or acquired immune deficiency conditions, potentially revealed by COVID-19, and must then be considered first when interpreting cell phenotyping values and when managing patients as well. ${ }^{15}$

\section{Conclusion}

For SARS-CoV-2 infection, alongside other clinical and biological parameters, the measurement of inflammatory cytokines, mainly IL-6, as well as CD4+ and CD8+ T cell assessment, should be systematically planned during management of the disease. These markers could be useful for identifying severe cases requiring prompt admission to intensive care units, and for monitoring patient disease progression. These investigations are within the reach of almost all clinical immunology laboratories in the world. Close collaboration between immunologists and physicians is essential for effective global efforts against this highly threatening pandemic.

\section{Acknowledgements}

We would like to express our sincere gratitude and deepest appreciation to Yacine Berka for his highly valuable contribution in the editing of this manuscript.

\section{Competing interests}

The authors have declared that no competing interests exist.

\section{Authors' contributions}

All authors contributed equally to this work. B.A. conceptualised and wrote the major parts of the manuscript. A.H. co-wrote the clinical aspects of the manuscript. M.A.S. contributed to the conceptualisation and validated a writing review of the manuscript.

\section{Ethical considerations}

Ethical clearance was not required for this study.

\section{Sources of support}

This research received no specific grant from any funding agency in the public commercial or not-for-profit sectors.

\section{Data availability statement}

Data sharing is not applicable to this article as no new data were created or analysed in this study. 


\section{Disclaimer}

The views and opinions expressed in this article are those of the authors and do not necessarily reflect the official policy or position of any affiliated agency of the authors.

\section{References}

1. Huang C, Wang Y, Li X, et al. Clinical features of patients infected with 2019 novel coronavirus in Wuhan, China. Lancet. 2020;395(10223):497-506. https://doi. org/10.1016/S0140-6736(20)30183-5

2. Xu B, Fan $C-Y$, Wang $A-L$, et al. Miao. Suppressed T cell-mediated immunity in patients with COVID-19: A clinical retrospective study in Wuhan, China. J Infect. 81(1):e51-e60.

3. Gao Y, Li T, Han M, et al. Diagnostic utility of clinical laboratory data determinations for patients with the severe COVID-19. J Med Virol. 2020;92(7):791-796. https:// doi.org/10.1002/jmv. 25770

4. Pedersen S-F, Ho Y-C. SARS-CoV-2: A storm is raging. J Clin Invest. 2020;130(5): 2202-2205. https://doi.org/10.1172/JCl137647

5. Henry B-M, De Oliveira M-H-S, Benoit S, et al. Hematologic, biochemical and immune biomarker abnormalities associated with severe illness and mortality in Coronavirus Disease 2019 (COVID-19): A meta-analysis. Clin Chem Lab Med. 2020;58(7):1021-1028. https://doi.org/10.1515/cclm-2020-0369

6. Liu J, Li S, Liu J, et al. Longitudinal characteristics of lymphocyte responses and cytokine profiles in the peripheral blood of SARS-CoV-2 infected patients. EBio Med. 2020;55:102763. https://doi.org/10.1016/j.ebiom.2020.102763
7. Zhou J, Chu H., Li C, et al. Active replication of Middle East respiratory syndrome coronavirus and aberrant induction of inflammatory cytokines and chemokines in human macrophages: Implications for pathogenesis. J Infect Dis 2014;209(9): 1331-1342. https://doi.org/10.1093/infdis/jit504

8. Diao B, Wang C, Tan $Y$, et al. Reduction and functional exhaustion of T cells in patients with coronavirus disease. Front. Immunol. 2020;11:827. https://doi. org/10.3389/fimmu.2020.00827

9. Zhu Z, Cai T, Fan L, et al. Clinical value of immune-inflammatory parameters to assess the severity of coronavirus disease 2019. Int J Infect Dis. 2020;95:332-339. https://doi.org/10.1016/j.ijid.2020.04.041

10. Qin C, Zhou L, Hu Z, et al. Dysregulation of immune response in patients with COVID-19 in Wuhan, China. Clin Infect Dis. 2020. https://doi.org/10.1093/cid/ ciaa248

11. Tan L, Wang Q, Zhang D, et al. Lymphopenia predicts disease severity of COVID-19: A descriptive and predictive study. Signal Transduct Target Ther. 2020;5(1):33 https://doi.org/10.1038/s41392-020-0159-1

12. $\mathrm{Xu} \mathrm{H}$, Zhong L, Deng J, et al. High expression of ACE2 receptor of 2019-nCoV on the epithelial cells of oral mucosa. Int J Oral Sci. 2020;12:8. https://doi.org/10.1038/ s41368-020-0074-x

13. Chen G, Wu D, Guo W, et al. Clinical and immunologic features in severe and moderate coronavirus disease 2019. J Clin Invest. 2020;130(5):2620-2629. https://doi.org/10.1172/JCl137244

14. Medeiros NI, Gomes JAS. Cytometric Bead Array [CBA] for measuring cytokine levels in Chagas disease patients. Methods Mol Biol. 2019;1955:309-314. https:// doi.org/10.1007/978-1-4939-9148-8_23

15. Brough H-A, Kalayci O, Sediva A, et al. Managing childhood allergies and immunodeficiencies during respiratory virus epidemics - The 2020 COVID-19 pandemic. Pediatr Allergy Immunol. 2020;31(5):442-448. https://doi.org/10.1111/ pai.13262 